presence of a bispecific antibody (BiAb) cross-linking the receptor with a tumor-associated antigen. While we could show efficacy of the SAR platform in different models, limited infiltration and immune suppression still hamper its function. We could previously demonstrate that $\mathrm{T}$ cell infiltration can be enhanced by transduction with carefully chosen chemokine receptors like CXCR6, CCR4 and CCR8. At the same time, gene silencing of checkpoint molecules like PD-1 can make $\mathrm{T}$ cells more resistant to immunosuppression, thus we assumed that combining these approaches might generate a desired $\mathrm{T}$ cell product.

Materials and Methods All constructs had been generated previously by overlap-extension cloning. The EGFRvIII (E3) SAR consists of extracellular EGFRvIII, transmembrane CD28 and intracellular CD28 and CD3ל. Human CXCR6-GFP, CCR4GFP and CCR8-GFP are composed of the chemokine receptors fused to GFP via a $2 \mathrm{~A}$ sequence. Primary human T cells were retrovirally transduced to stably express the SAR and chemokine receptors. We analyzed migration, cytotoxicity and activation of the single and double (E3 SAR and chemokine receptor) transduced $\mathrm{T}$ cells. In addition, PD-1 was knocked out using CRISPR-Cas9 and killing kinetics of target cells and $\mathrm{T}$ cell activation were assessed.

Results Co-transduction with chemokine receptors significantly increased migration of E3 SAR $\mathrm{T}$ cells to their respective ligand while lysis of target-expressing tumor cell and $\mathrm{T}$ cell activation in the presence of $\mathrm{BiAb}$ were not affected in vitro. Additionally knocking out PD-1 enhanced killing kinetics and activation of E3 SAR and E3 SAR + CXCR6-GFP transduced $\mathrm{T}$ cells compared to corresponding mock electroporated $\mathrm{T}$ cells.

Conclusions Using the controllable and modular SAR - BiAb platform SAR $\mathrm{T}$ cell activation can be limited by stopping $\mathrm{BiAb}$ dosing if adverse events occur. In addition, SAR $\mathrm{T}$ cells can be redirected to an alternative tumor-associated antigen by exchanging the $\mathrm{BiAb}$ in the case of antigen escape. Here we present add-ons to this approach for increased tumor infiltration and resistance to immunosuppression. Since migration is enhanced upon co-transduction with chemokine receptors and target cell lysis is accelerated upon PD-1 knockout in vitro these two additional modifications seem very promising options to further improve tumor control in vivo.

Disclosure Information M. Schwerdtfeger: None. M. Benmebarek: None. F. Märkl: None. C.H. Karches: A. Employment (full or part-time); Significant; Daiichi Sankyo Deutschland GmbH. A. Öner: None. M. Geiger: A. Employment (full or part-time); Significant; Roche. B. Cadilha: None. S. Endres: None. V. Desiderio: None. C. Klein: A. Employment (full or part-time); Significant; Roche. S. Kobold: None.

\section{P06.07 IN VIVO STUDIES OF IMMUNOMODULATORY A-CTLA-4 ANTIBODY IN A HUMANIZED MOUSE MODEL}

C Reitinger*, F Nimmerjahn. University Erlangen, Erlangen, Germany

\subsection{6/jitc-2021-ITOC8.41}

Background Recent findings in cancer immunotherapy have reinforced the hypothesis that the immune system is able to control most cancers. Immunomodulatory antibodies can enhance immune responses, having the potential to generate anti-cancer immunity. ${ }^{1-4}$

Materials and Methods Most current studies addressing this question are performed in murine mouse model systems or use in vitro culture systems, which do not reflect the human in vivo situation, potentially leading to results that cannot be fully translated into human cancer therapy. Therefore, it is necessary to establish a new mouse model, which allows the study of cancer immunotherapy in the context of a human immune system. We focused on the establishment of a humanized mouse model, in which different immunomodulatory antibodies can be tested in the presence of a human immune system.

Results First experiments concerning the suitability to test immunomodulatory antibodies in the humanized mouse model, revealed that effects of checkpoint-control antibody a-CTLA-4 were similar to the effects seen in patients of clinical studies. To analyse the anti-tumor activities of immunomodulatory antibodies in vivo we are establishing a human melanoma-like tumor model in humanized mice.

Conclusions This enables us to test the efficacy of immunomodulatory agonistic antibodies (such as CP-870,893) and checkpoint control antibodies (such as anti-CTLA-4) in eliminating a melanoma-like tumor. Furthermore, parameters like tumor infiltrating human cells und cytokine/chemokine production can be analysed.

\section{REFERENCES}

1. Schuster $M$, Nechansky $A$, Loibner $H$. Cancer immunotherapy. Biotechnol J 2006;1:138-147.

2. Mellman I, Coukos G, Dranoff G. Cancer immunotherapy comes of age. Nature rev 2011;480:480-489.

3. Finn OJ. Immuno-oncology: understanding the function and dysfunction of the immune system in cancer. Annals of Oncology 2012;23:vii6-vii9.

4. Langer LF, Clay TM, Morse MA. Update on anti-CTLA-4 in clinical trials. Expert Opin Biol Ther 2007;8:1245-1256.

Disclosure Information C. Reitinger: None. F. Nimmerjahn: None.

\section{P07 Cell therapy in haematologic diseases}

\section{P07.01 A MODULAR AND CONTROLLABLE T CELL THERAPY PLATFORM FOR AML}

${ }^{1} \mathrm{M}$ Benmebarek*, 'B Loureiro Cadilha, ${ }^{2} \mathrm{M}$ Herrmann, ${ }^{3} \mathrm{~S}$ Schmitt, ${ }^{1} \mathrm{~S}$ Lesch, ${ }^{1} \mathrm{~S}$ Stoiber ${ }^{4} \mathrm{~A}$ Darwich, ${ }^{3} \mathrm{C}$ Augsberger, ${ }^{3} \mathrm{~B}$ Brauchle, ${ }^{1} \mathrm{M}$ Schwerdtfeger, ${ }^{1} \mathrm{~A}$ Gottschlich, ${ }^{1}$ Rataj, ${ }^{3} \mathrm{NC}$ Fenn, ${ }^{5} \mathrm{C}$ Klein, ${ }^{3} \mathrm{M}$ Subklewe, ${ }^{1} \mathrm{~S}$ Endres, ${ }^{6} \mathrm{~K}$ Hopfner, ${ }^{1} \mathrm{~S}$ Kobold. ${ }^{1} \mathrm{Center}$ of Integrated Protein Science Munich (CIPS-M) and Division of Clinical Pharmacology, Munich, Germany; ${ }^{2}$ Department of Medicine III, Klinikum der Universität München, LMU, Munich, Germany; ${ }^{3}$ Department of Medicine III, Klinikum der Universität München, Munich, Germany; ${ }^{4}$ Mucosal Immunology and Microbiota Lab, Humanitas Clinical and Research Center, Milan, Italy; ${ }^{5}$ Roche Innovation Center Zurich, Schlieren, Switzerland; ${ }^{6}$ Gene Center, LMU, Munich, Germany

\subsection{6/jitc-2021-ITOC8.42}

Background Targeted immunotherapies have shown limited success in the context of acute myeloid leukemia (AML). The mutational landscape, heterogeneity attributed to this malignancy and toxicities associated with the targeting of myeloid lineage antigens, it has become apparent that a modular and controllable cell therapy approach with the potential to target multiple antigens is required. We propose a controlled ACT 
approach, where $\mathrm{T}$ cells are equipped with synthetic agonistic receptors (SARs) that are selectively activated only in the presence of a target AML-associated antigen, and a cross-linking tandem single chain variable fragment $(\mathrm{taFv})$ specific for both (SAR) T cell and tumour cell.

Materials and Methods A SAR composed of an extracellular EGFRvIII, trans- membrane CD28, and intracellular CD28 and $\mathrm{CD} 3 \mathrm{z}$ domains was fused via overlap- extension PCR cloning. T cells were retrovirally transduced to stably express our SAR construct. SAR-specific taFvs that target AML-associated antigens were designed and expressed in Expi293 $\mathrm{F}^{\mathrm{TM}}$ cells and purified by nickel affinity and size exclusion chromatography (SEC). We validated our approach in three human cancer models and patient-derived AML blasts expressing our AML-associated target antigens CD33 and CD123.

Results Anti-CD33-EGFRvIII and anti-CD123 EGFRvIII taFv, monovalently selective for our SAR, induced conditional antigen-dependent activation, proliferation and differentiation of SAR-T cells. Further, SAR T cells bridged to their target cells by taFv could form functional immunological synapses, resulting in efficient tumor cell lysis with specificity towards CD33-expressing AML cells. SAR-taFv combination could also mediate specific cytotoxicity against patientderived AML blasts and leukemic stem cells whilst driving SAR $\mathrm{T}$ cell activation. In vivo, treatment with SAR-taFv combination could efficiently eradicate leukemia and enhance survival in an AML xenograft models. Furthermore, we could show selective activation of SAR T cells, as well as a controllable reversibility and modularity of said activation upon depletion of the $\mathrm{T}$ cell engaging molecule, both in vitro and in vivo.

Conclusions Here we apply the SAR-taFv platform in efforts to deliver specific and conditional activation of SAR-transduced $\mathrm{T}$ cells, and targeted tumour cell lysis. The modularity of our platform will allow for a multi-targeting ACT approach with the potential to translate the ACT successes of B cell malignancies to AML. With a lack of truly specific AML antigens, it is invaluable that this approach possesses an intrinsic safety switch via its taFv facet. Moreover, we are able to circumvent pan-T cell activation due to the specific targeting and activation of SAR T cells.

Disclosure Information M. Benmebarek: None. B. Loureiro Cadilha: None. M. Herrmann: None. S. Schmitt: None. S. Lesch: None. S. Stoiber: None. A. Darwich: None. C. Augsberger: None. B. Brauchle: None. M. Schwerdtfeger: None. A. Gottschlich: None. A. Gottschlich Rataj: None. N.C. Fenn: None. C. Klein: None. M. Subklewe: None. S. Endres: None. K. Hopfner: None. S. Kobold: None.

\section{P07.02 REGULATION OF CD19 CAR T- CELL ACTIVATION BASED ON ENGINEERED NUCLEAR FACTOR OF ACTIVATED T CELLS ARTIFICIAL TRANSCRIPTION FACTORS}

${ }^{1} \mathrm{D}$ Lainšček*, ${ }^{2} \mathrm{~V}$ Mikolič, ${ }^{1} \mathrm{~S}^{2}$ Malenšek, ${ }^{1} \mathrm{~A}$ Verbič, ${ }^{1} \mathrm{R}$ Jerala. ${ }^{1}$ National institute of Chemistry, Ljubljana, Slovenia; ${ }^{2}$ University Medical Centre Ljubljana, Ljubljana, Slovenia

\subsection{6/jitc-2021-ITOC8.43}

Background CD19 CAR T- cells (Chimeric antigen receptor T cells that recognize CD19) present a therapeutic option for various malignant diseases based on their ability to specifically recognize the selected tumour surface markers, triggering immune cell activation and cytokine production that results in killing cancerous cell expressing specific surface markers recognized by the CAR. The main therapeutic effect of CAR is a specific $T$ cell activation of adequate cell number with sequential destruction of tumorous cells in a safe therapeutic manner. In order to increase $\mathrm{T}$ cell activation, different activation domains were introduced into CAR. CAR T-cells are highly efficient in tumour cell destruction, but may cause serious side effects that can also result in patient death so their activity needs to be carefully controlled. ${ }^{1}$ Several attempts were made to influence the CAR T cell proliferation and their activation by adding $\mathrm{T}$ cell growth factors, such as IL-2, into patients, however this approach of increasing the number of activating $\mathrm{T}$ cells with no external control over their number can again lead to non-optimal therapeutic effects. Different improvements were made by designing synthetic receptors or small molecule-inducible systems etc., which influence regulated expansion and survival of CAR T cells. $^{2}$

Material and Methods In order to regulate CD19 CAR-T cell activity, different NFAT2 based artificial transcription factors were prepared. The full length NFAT2, one of the main players in T cell IL2 production, a key cytokine for $\mathrm{T}$ cell activation and proliferation was truncated by deletion of its own activation domain. Next, we joined via Gibson assembly tNFAT2 2 1-593 coding sequence with domains of different heterodimerization systems that interact upon adding the inductor of heterodimerization. The interaction counterparts were fused to a strong tripartite transcriptional activator domain VPR and/or strong repressor domain KRAB resulting in formation of an engineered NFAT artificial transcription (NFAT-TF) factors with external control. To determine the activity of NFAT-TF HEK293, Jurkat or human T cells were used.

Results Based on luciferase assay, carried out on NFAT-TF transfected HEK293 cells we first established that upon adding the external inductor of heterodimerization, efficient gene regulation occurs, according to VPR or KRAB domain appropriate functions. Findings were then transferred to Jurkat cells that were electroporated with appropriate DNA constructs, coding for NFAT-TF and CD19 CAR. After Raji:Jurkat co-culture ELISA measurements revealed that IL2 production and therefore CD19 CAR-T cell activity can be controlled by the action of NFAT-TF. The same regulation over the activity and subsequent proliferation status was also observed in retrovirally transduced human T-cells.

Conclusion We developed a regulatory system for therapeutic effect of CD19 CAR-T cells, a unique mechanism to control T cell activation and proliferation based on the engineered NFAT2 artificial transcription factor.

\section{REFERENCES}

1. Bonifant $\mathrm{CL}$, et al. Toxicity and management in CAR T-cell therapy. Mol Ther Oncolytics 2016;3:16011.

2. Wu C-Y, et al. Remote control of therapeutic $T$ cells through a small moleculegated chimeric receptor. Science 2015;80:350.

Disclosure Information D. Lainšček: None. V. Mikolič: None. Š. Malenšek: None. A. Verbič: None. R. Jerala: None. 